\title{
Investigation of the effects of intravenous magnesium sulphate on cardiac rhythm in acute myocardial infarction
}

\author{
C Roffe, S Fletcher, K L Woods
}

\begin{abstract}
Objective-To examine the effect of doubling serum magnesium concentration on the incidence of arrhythmias in patients with suspected acute myocardial infarction.

Design-Randomised double blind clinical trial.

Setting-Coronary care unit of a teaching hospital.

Patients-Clinical data were collected on 2316 randomised patients with suspected acute myocardial infarction. Holter monitoring was performed in a subgroup of 70 patients and analysed in 48 patients in whom acute myocardial infarction was
\end{abstract} confirmed.

Interventions-By random allocation, patients received either an intravenous loading dose of $8 \mathrm{mmol}$ magnesium sulphate over five minutes plus $65 \mathrm{mmol}$ over the next 24 hours, or equal volumes of saline.

Main outcome measures-(a) Clinically documented arrhythmias; (b) use of antiarrhythmic treatments, cardioversion, and insertion of a pacemaker; (c) incidence of all abnormal rhythms during Holter monitoring.

Results-In the main trial the incidence of rhythm disturbance while in the coronary care unit (expressed as the odds ratio (OR) for magnesium: placebo and its $95 \%$ confidence interval) was not significantly different between treatment groups for ventricular fibrillation (OR $0.74 ; 0.46$ to 1.20 ), ventricular tachycardia (OR 0.87 ; 0.63 to $1 \cdot 20$ ), supraventricular tachycardia (OR $0.69 ; 0.38$ to 1.26 ), atrial fibrillation (OR $0.92 ; 0.69$ to $1 \cdot 23$ ), or heart block of any degree (OR 1.17; 0.83 to $1 \cdot 65$ ). Sinus bradycardia was significantly more common in the magnesium group (OR $1.38 ; 1.03$ to $1.85 ; p=$ $0 \cdot 02$ ). These findings were corroborated by the use of treatments for rhythm disturbance and the data from Holter monitoring.

Conclusion-The regimen of intravenous magnesium sulphate used here had no significant effect on arrhythmia in acute myocardial infarction. The reduction in mortality that has been shown with this form of treatment is not attributable to suppression of life threatening rhythm disturbances.

(Br Heart f 1994;71:141-145)
Intravenous magnesium salts have been used as antiarrhythmic agents for over 50 years ${ }^{1}$ and have been given (but never rigorously evaluated) in a wide range of rhythm disorders. In recent years there has been particular interest in their possible prophylactic value in reducing the frequency of arrhythmic complications of acute myocardial infarction. Experimentally, intravenous magnesium sulphate reduced the incidence of ventricular fibrillation after coronary ligation in dogs. ${ }^{23}$ A concentration dependent suppression of ventricular arrhythmias occurred over the range of $1.2-4.8 \mathrm{mM}\left[\mathrm{Mg}^{2+}\right]$ during reperfusion of the isolated rat heart after temporary left main coronary artery occlusion ${ }^{4}$; suppression of ligation induced (but not reperfusion induced) ventricular fibrillation in the same rat model had earlier been reported at $7.3 \mathrm{mM}\left[\mathrm{Mg}^{2+}\right] .^{5}$ In the isolated guinea pig myocyte depolarised by high external $\left[\mathrm{K}^{+}\right], 4 \mathrm{mM}$ external $\left[\mathrm{Mg}^{2+}\right]$ significantly increased the threshold concentration of isoprenaline required to produce automaticity. ${ }^{6}$

Several small clinical trials of intravenous magnesium sulphate or chloride in acute myocardial infarction have given mixed results. Attained $\left[\mathrm{Mg}^{2+}\right]$ has been substantially lower than in the experimental studies described earlier, in the range $1.0-1.5 \mathrm{mmol} / 1$ (normal range approximately $0.70-0.95$ $\mathrm{mmol} / \mathrm{l}$ ). Four double blind, randomised trials in a total of nearly 900 patients with suspected or definite acute myocardial infarction showed no significant reduction in any class of ventricular arrhythmia. ${ }^{7-10}$ One study (48 patients with confirmed acute myocardial infarction) showed a highly significant reduction in incidence of ventricular tachycardia in the magnesium group but a higher incidence of other ventricular ar:hythmias. ${ }^{11}$ Two randomised trials, each of fewer than 50 patients, reported significant reductions in the incidence of ventricular arrhythmias. ${ }^{1213} \mathrm{~A}$ further randomised trial in which 130 patients with confirmed acute myocardial infarction received either intravenous magnesium chloride or placebo showed a highly significant reduction in the incidence of supraventricular tachycardias in the magnesium group but no effect on ventricular arrhythmias. ${ }^{14}$ Pooled analysis of the arrhythmia data in these trials is unhelpful because of the differing definitions of rhythm end points.

We have therefore analysed all available data on the frequency of arrhythmias in a large double blind trial (the second Leicester 
intravenous magnesium intervention trial LIMIT-2) study) in which 2316 patients with suspected acute myocardial infarction were randomly allocated to receive intravenous magnesium sulphate or saline for 24 hours after admission. In this study 28 day mortality was the primary endpoint and frequency of arrhythmia within the coronary care unit a secondary endpoint defined in the protocol. Three methods (each blind to treatment allocation) were used to identify a possible antiarrhythmic effect of magnesium: an analysis of all arrhythmias recorded clinically in trial patients during routine monitoring while in the coronary care unit; analysis of the use of antiarrhythmic treatments specified by the protocol; and a Holter monitoring study in a representative subgroup of trial patients to quantify all abnormal rhythms. A summary of the data from the first two analyses were presented in brief in the first trial report. ${ }^{15}$

\section{Patients and methods}

The protocol of LIMIT-2 and data on 28 day mortality and early morbidity have been described elsewhere. ${ }^{15}$ Briefly, consenting patients entering the coronary care unit of Leicester Royal Infirmary between September 1987 and February 1992 were eligible for randomisation if they were suspected of having acute myocardial infarction of less than 24 hours in duration. Exclusion criteria were complete heart block or serum creatinine $>300 \mu \mathrm{mol} / 1$. The trial treatment was 8 mmol magnesium sulphate infused intravenously over five minutes, then $65 \mathrm{mmol}$ over 24 hours. The placebo treatment was an equal volume of saline. The magnesium regimen raised serum $\left[\mathrm{Mg}^{2+}\right]$ to a mean of $1.5 \mathrm{mmol} / \mathrm{l}$ for the duration of treatment; the concentration then declined to the normal range during a further 24 hours. Trial treatments were supplied in identical coded packs and the allocation code was not broken until the end of the trial. Analysis was by intention to treat.

While in the coronary care unit (about 48 hours on average) all patients underwent continuous electrocardiographic monitoring. While in the unit, a standard clinical record is made for each patient (whether or not in a trial) of all significant events and of treatments given. These data are recorded by clinical staff during the admission and subsequently entered into a computer database. The occurrence of specific classes of arrhythmia is recorded but not quantified. Treatments are given according to a comprehensive, written management policy for the unit. Patients entering the LIMIT-2 study were managed conventionally in all other aspects of treatment. No antiarrhythmic agents are used prophylactically, with the exception of lignocaine given for 24 hours after emergency cardioversion for ventricular tachycardia.

Holter monitoring was performed for the first 24 hours after randomisation in a subgroup of 70 patients entering the trial. The diagnosis of acute myocardial infarction was subsequently confirmed in 51 of these, in three of whom the recordings were technically inadequate. The analysis was carried out on the remaining 48 patients with confirmed acute myocardial infarction, among whom the recording was incomplete for two patients in the placebo group because of a premature end when emergency cardioversion was given for ventricular fibrillation. These patients were included in the analysis of incidence of arrhythmia but could not be included in the analysis of frequency of events over 24 hours. Extrapolation from the available recordings from these two patients was considered to be invalid as there was a strong general trend to lower hourly rates of events over the recording period. Tapes were analysed by computer, with visual verification of all abnormal rhythms. All data were collected without knowledge of the patients' treatment group. Ventricular tachycardia was defined as the occurrence of $>$ six ventricular extra systoles at a rate $>100$ beats $/ \mathrm{min}$ and $>120 \%$ of the sinus rate. Runs not meeting these criteria were classified as salvos.

Hypotheses were tested by $\chi^{2}$ and MannWhitney statistics.

\section{Results}

The incidence of clinically documented tachycardias in the trial groups was not significantly different for ventricular fibrillation, ventricular tachycardia, supraventricular tachycardia, or atrial fibrillation (fig 1) although each was slightly less common in

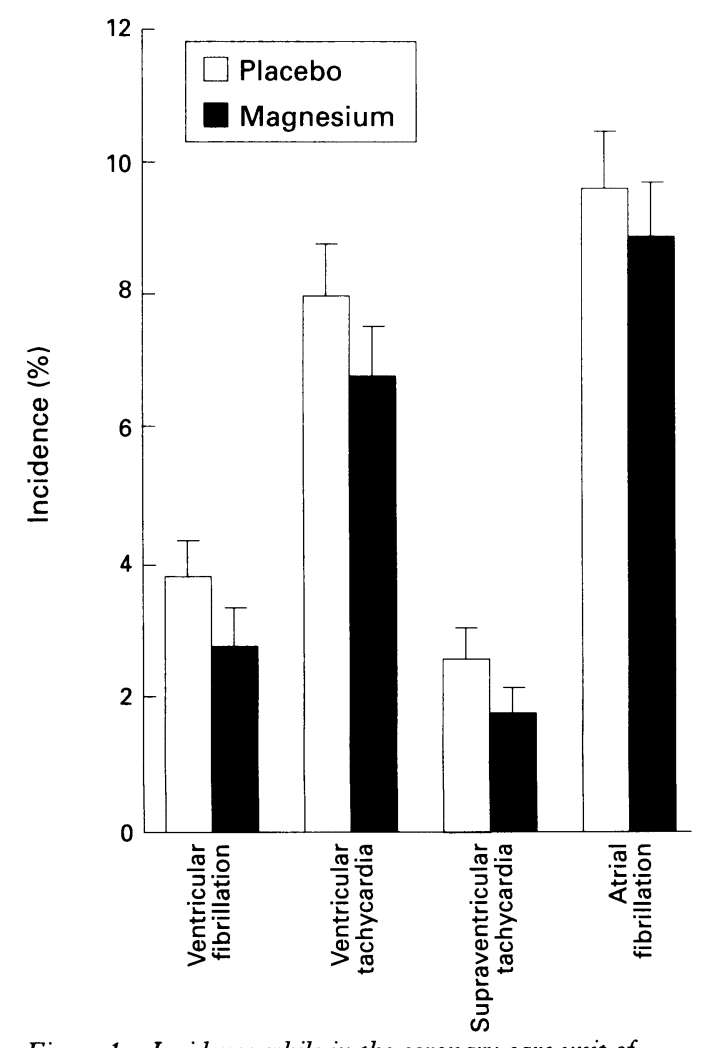

Figure 1 Incidence while in the coronary care unit of tachycardia in patients randomised to intravenous magnesium sulphate $(n=1159)$ or placebo $(n=1157)$. 
Table 1 Incidence of heart block and use of temporary pacing in randomised groups while in the coronary care unit

\begin{tabular}{llll}
\hline & $\begin{array}{l}\text { Magnesium } \\
(n=1159) \\
\%\end{array}$ & $\begin{array}{l}\text { Placebo } \\
(n=1157)\end{array}$ & \\
Degree of block & $2 \cdot 1$ & $1 \cdot 3$ & $p$ Value \\
\hline First & $2 \cdot 7$ & $2 \cdot 2$ & $0 \cdot 11$ \\
Second & $2 \cdot 1$ & $2 \cdot 5$ & 0.51 \\
Third & $4 \cdot 8$ & $4 \cdot 2$ & 0.57 \\
Any & $2 \cdot 2$ & $1 \cdot 6$ & $0 \cdot 37$ \\
Temporary pacemaker & $2 \cdot 2$ & & \\
\hline
\end{tabular}

Table 2 Odds ratios (magnesium: placebo) for incidence of arrhythmias and interventions $(n=2316)$

\begin{tabular}{lll}
\hline Arrhythmia or intervention & Odds ratio $(95 \% \mathrm{Cl})$ & p Value \\
\hline Ventricular tachycardia & $0.87(0.63$ to 1.20$)$ & NS \\
Ventricular fibrillation & $0.74(0.46$ to 1.20$)$ & NS \\
Supraventricular tachycardia & $0.69(0.38$ to 1.26$)$ & NS \\
Atrial fibrillation & $0.92(0.69$ to 1.23$)$ & NS \\
Any tachycardia & $0.82(0.66$ to 1.02$)$ & 0.06 \\
Lignocaine & $0.76(0.51$ to 1.11$)$ & NS \\
Amiodarone & $0.74(0.49$ to 1.10$)$ & NS \\
Digoxin & $0.93(0.66$ to 1.31$)$ & NS \\
DC cardioversion & $1.02(0.66$ to 1.57$)$ & NS \\
Heart block (any degree) & $1.17(0.83$ to 1.65$)$ & NS \\
Sinus bradycardia & $1.38(1.03$ to 1.85$)$ & 0.02 \\
Atropine & $1.69(1.25$ to 2.30$)$ & $<0.001$ \\
Temporary pacemaker & $1.32(0.70$ to 2.51$)$ & NS \\
\hline
\end{tabular}

the magnesium group. Heart block, whether analysed by grade of block or pooling all grades, was of similar incidence in both groups (table 1). The only difference in cardiac rhythm that reached significance between the groups was the documentation of sinus bradycardia, which was recorded in $10.8 \%$ of the magnesium group and $8.0 \%$ of the placebo group $(p=0.02)$. Table 2 shows the effects as odds ratios (ORs) and their $95 \%$ confidence intervals ( $95 \%$ CIs).

These findings are supported by the data on treatments used and procedures performed. There was a slightly lower incidence of the use of lignocaine, amiodarone, and digoxin in the magnesium group but none of the comparisons reached significance (fig 2).

Figure 2 Use of treatments for tachycardia in the randomised groups of LIMIT-2.
Table 3 Characteristics of patients included in the Holter monitoring subgroup

\begin{tabular}{lcc}
\hline & $\begin{array}{c}\text { Magnesium } \\
(n=22)\end{array}$ & $\begin{array}{l}\text { Placebo } \\
(n=26)\end{array}$ \\
\hline Age (yr, SD) & $65 \cdot 7(12 \cdot 7)$ & $60 \cdot 2(9 \cdot 7)$ \\
Men (\%) & $15(68 \cdot 2)$ & $19(73 \cdot 1)$ \\
Site of infarction (\%): & $12(54 \cdot 5)$ & $14(53 \cdot 8)$ \\
$\quad$ Anterior & $7(31 \cdot 5)$ & $10(38 \cdot 5)$ \\
$\quad$ Inferior & $3(13 \cdot 6)$ & $2(7 \cdot 6)$ \\
$\quad$ Indeterminate & $4 \cdot 2$ & $3 \cdot 5$ \\
$\begin{array}{l}\text { Mean delay from onset } \\
\text { to admission (h) }\end{array}$ & & \\
Peak creatine kinase (u/l, SD) & $2438(1773)$ & $1937(1469)$ \\
Thrombolysis (\%) & $14(63 \cdot 6)$ & $17(65 \cdot 3)$ \\
\hline
\end{tabular}

Insertion of a temporary pacemaker and direct current cardioversion were similar in the two groups. Consistent with the significant excess of sinus bradycardia in the magnesium group, there was a correspondingly higher rate of use of atropine $(p<0.001)$. A detailed analysis of mortality and morbidity in the trial shows no evidence that the occurrence of sinus bradycardia was deleterious ${ }^{15}$; left ventricular failure in the coronary care unit and 28 day mortality were both significantly lower in the magnesium group.

Among the analysable patients included in the Holter monitoring study who had a confirmed acute myocardial infarction, 22 received magnesium sulphate and 26 placebo. Table 3 shows their clinical characteristics. The incidence of the various classes of disturbance of ventricular rhythm detected during Holter monitoring (fig 3) was not significantly different in the two groups. Because the numbers of events in each class were not normally

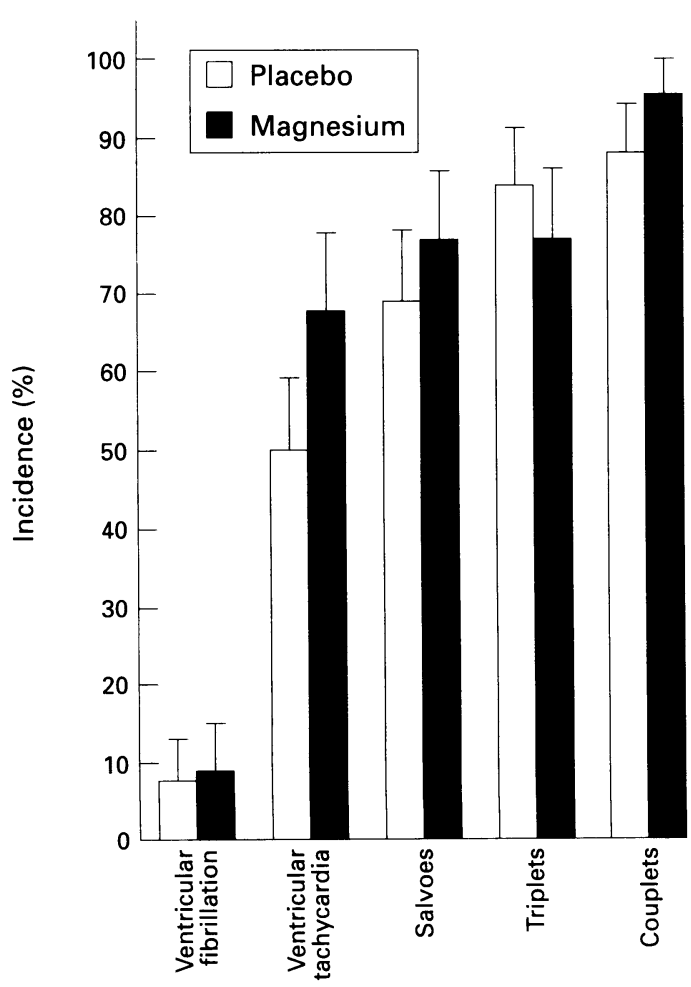

Figure 3 Cumulative incidence of ventricular rhythm disturbances during 24 hour Holter monitoring among patients with confirmed myocardial infarction randomised to intravenous magnesium sulphate $(n=22)$ or placebo $(n=24)$. 
Table 4 Median number of ventricular arrhythmias $n / 24 h$ (two recordings were incomplete in the placebo group and were excluded)

\begin{tabular}{lccl}
\hline & $\begin{array}{c}\text { Magnesium } \\
(n=22)\end{array}$ & $\begin{array}{c}\text { Placebo } \\
(n=24)\end{array}$ & $p$ Value \\
\hline Salvos & 4.0 & 2.5 & 0.73 \\
Triplets & 5.5 & 3.5 & 0.99 \\
Couplets & 21.0 & 13.5 & 0.43 \\
Ventricular extrasystoles & 879 & 512 & 0.23 \\
\hline
\end{tabular}

distributed, they were summarised (table 4) by their median values and comparisons were tested by a non-parametric method (MannWhitney test). No significant differences were found in the amount of any class of ventricular arrhythmia that occurred during the 24 hours of recording.

\section{Discussion}

The LIMIT-2 study is by far the largest randomised trial in which the possible prophylactic value of an intravenous magnesium salt against ischaemic arrhythmias has been examined. Although the identification of arrhythmias from routine continuous monitoring is known to be incomplete, the likelihood of detection is in proportion to the clinical significance of the arrhythmia. Moreover, the double blind design of the protocol and the corroboration provided by the analysis of antiarrhythmic treatments given support the validity of the findings from the main study. The Holter monitoring study in a representative group of patients with confirmed myocardial infarction, designed to achieve complete and accurate ascertainment of all disturbances of rhythm, also showed no significant antiarrhythmic effect of magnesium.

The apparent discrepancy between the findings of our study and the experimental models of myocardial ischaemia ${ }^{2-6}$ may reflect the difference in the external $\left[\mathrm{Mg}^{2+}\right]$ reached or may be model dependent. The published clinical trials of magnesium salts in myocardial infarction have generally been small and their findings inconsistent. The inconsistency may partly arise from the retrospective analysis of many different classes and measures of arrhythmias. It is a strength of the present investigation that the categories of arrhythmia analysed were either defined in advance in the clinical data system (the main study) or else were comprehensive and mutually exclusive classes describing the totality of abnormal rhythms occurring during the study period (the Holter study). Data derived significance testing was therefore precluded.

Outside the context of acute myocardial infarction, intravenous magnesium salts have been reported to be variably effective in a wide range of arrhythmias, including sustained monomorphic ventricular tachycardia, ${ }^{16}$ polymorphic ventricular tachycardia (torsade de pointes) of various aetiologies, ${ }^{17}$ re-entrant supraventricular tachycardia, ${ }^{18-20}$ and multifocal atrial tachycardia. ${ }^{21}$ In none of these have randomised trials of efficacy been carried out. Torsade de pointes is the arrhythmia that seems to be most consistently responsive to intravenous magnesium. The mechanism of action does not depend on preexisting hypomagnesaemia or on shortening of an abnormally prolonged QT interval. The effects of doubling of serum $\left[\mathrm{Mg}^{2+}\right]$ on generation and propagation of the cardiac action potential have been examined in several clinical electrophysiological studies. ${ }^{22-25}$ There is a small but consistent prolongation of the atrium-His interval due to slowing of conduction through the upper AV node. This action may account for the reported efficacy of magnesium salts in the treatment of AV re-entrant supraventricular tachycardia; it seems to arise from competitive blockade of the slow $\mathrm{Ca}^{2}+$ channel and resembles the action of the organic calcium antagonist verapamil.

Because of the reported effect of $\mathrm{Mg}^{2+}$ on conduction through the AV node, evidence was sought in our study for any association between magnesium treatment and the development or exacerbation of heart block; none was found. The increased incidence of sinus bradycardia was not predicted by electrophysiological studies of intravenous $\mathbf{M g}^{2+}$ in humans, which have indicated a lack of effect on the length of the sinus node cycle. ${ }^{22-2426}$ In these studies a direct inhibition of the sinus node by $\mathrm{Mg}^{2}+$ through an effect on the slow $\mathrm{Ca}^{2}+$ channel or the acetylcholine activated $\mathrm{K}^{+}$channel $^{27}$ might have been offset by the reflex response to peripheral vasodilatation induced by $\mathrm{Mg}^{2+}$, but if so it is unclear why our group of patients should have responded differently. A more likely explanation is that the relatively small electrophysiological studies lacked the statistical power to detect a modest $\mathbf{M g}^{2}+$ effect.

These findings cannot be generalised to patients with hypomagnesaemia, such as commonly occurs during cardiopulmonary bypass; a randomised placebo controlled trial has shown that partial repletion of $\left[\mathrm{Mg}^{2+}\right]$ with intravenous magnesium sulphate significantly reduces the incidence of postoperative ventricular arrhythmias in this situation. ${ }^{28}$ Further systematic study of magnesium salts is required to clarify the extensive case reports suggesting efficacy against a variety of arrhythmias. In torsades de pointes, for example, suppression of early afterdepolarisations and triggered activity by extracellular $\left[\mathrm{Mg}^{2+}\right]$ offers a cellular mechanism in support of the empirical clinical findings. ${ }^{29}$ In acute myocardial infarction, however, we have found no evidence that doubling serum $\left[\mathrm{Mg}^{2+}\right]$ reduces the incidence of early arrhythmias. The significant reduction of mortality seen in LIMIT-2 and in the pooled analysis of earlier studies ${ }^{15}$ cannot be accounted for by prevention of lethal arrhythmias.

The LIMIT-2 study was supported by project grants from the Leicestershire Health Authority and the British Heart Foundation. We thank the staff of the coronary care unit of the Leicester Royal Infirmary for their help in the collection of data, and the staff of the electroca
their help with the Holter study. 
1 Boyd L, Scherf D. Magnesium sulfate in paroxysmal tachycardia. $A m \mathcal{F}$ Med Sci 1943;206:43-8.

2 Barros L, Da-Luz P, Siveira M, Chagas A, Pileggi F. Ventricular fibrillation in acute experimental myocardial ischaemia: protection by magnesium sulphate. Braz $f$ ischaemia: protection by magr

3 Billman G, Hoskins R. Prevention of ventricular fibrilla tion with magnesium sulphate. Eur $\mathcal{F}$ Pharmacol 1988 158:167-7

4 Bril A, Rochette L. Prevention of reperfusion-induced ventricular arrhythmias in isolated rat heart with magnesium. Can f Physiol Pharmacol 1990;68:694-9.

5 Crampton $\mathrm{R}$, Clark $\mathrm{C}$. Varying extracellular $\left[\mathrm{Mg}^{+}\right]$alters ischaemic and reperfusion ventricular tachyarrhythmias. Circulation 1983;68(supp 111):146.

6 Hasegawa J, Matsumoto T, Takami T, Fujimoto Y, Kotake $H$, Mashiba $H$. Suppression of catecholamineinduced abnormal pacemaker activities by magnesium pacesium ion in guinea

7 Morton B, Nair R, Smith F, McKibbon T, Poznanski W. Magnesium therapy in acute myocardial infarctionMagnesium therapy in acute myocardial infarctid

8 Smith LF, Heagarty AM, Bing RF, Barnett DB. Intravenous infusion of magnesium sulphate after acute myocardial infarction: effects on arrhythmias and mortality. Int $\mathcal{F}$ Cardiol 1986;12:175-80.

9 Feldstedt M, Boesgaard S, Bouchelouche P, et al. Magnesium substitution in acute ischaemic heart syndromes. Eur Heart $\mathcal{F}$ 1991;12:1215-8.

10 Shechter M, Hod H, Marks N, Behar S, Kaplinsky E, Rabinowitz B. Beneficial effect of magnesium sulfate in acute myocardial infarction. Am $\mathcal{F}$ Cardiol 1990;66: $271-4$

11 Ceremuzynski L, Jurgiel R, Kulakowski P, Gebalska J. Threatening arrhythmias in acute myocardial infarction are prevented by intravenous magnesium sulphate. Am Heart $\mathcal{F} 1989 ; 118: 1333-4$.

12 Berschat $F$, Ising $H$, Gunther $T$, Sorgenfrei J, Wollitz $M$, Ibe $\mathrm{K}$. Antiarrhythmic effects of magnesium infusions in Ibe $\mathrm{K}$. Antiarrhythmic effects of magnesium infusions in patients with acute my

13 Abraham AS, Rosenmann D, Kramer M, et al. Magnesium in the prevention of lethal arrhythmias in acute myocardial infarction. Arch Intern Med 1987; 147:753-5.

14 Rasmussen $H$, Suenson $M$, Norregard P, Balslev S. Magnesium infusion reduces the incidence of arrhythmias in acute myocardial infarction. A double-blind placebo-controlled study. Clin Cardiol 1987;10:351-6.

15 Woods KL, Fletcher S, Roffe C, Haider Y. Intravenous magnesium sulphate in suspected acute myocardial infarction: results of the second Leicester Intravenous
Magnesium Intervention Trial (LIMIT-2), Lancet 1992;339:1553-8.

16 Allen B, Brodsky M, Capparelli E, Luckett C, Iseri L Magnesium suiphate therapy for sustained monomorphic Magnesium sulphate therapy for sustained monomorph

17 Tzivoni D, Banai S, Schuger C, et al. Treatment of torsade de pointes with magnesium sulphate. Circulation 1988;77:392-7.

18 Etienne Y, Blanc J, Boschat J, et al. Effets antiarythmiques du sulphate de magnesium intraveineux dans les tachycardies supraventriculaires paroxystiques. Ann Cardiol Angeiol 1988;37:535-8.

19 Wesley R, Haines D, Lermon B, DiMarco J, Crampton R. Effect of intravenous magnesium sulphate on supraventricular tachycardia. Am $\mathcal{f}$ Cardiol 1989;63:1129-31.

20 Sager P, Widerhorn J, Petersen R, et al. Prospective evaluation of parenteral magnesium sulphate in the treatment of patients with reentrant $\mathrm{AV}$ supraventricular tachycardia. Am Heart ₹ 1990;119:308-16.

21 Iseri L, Fairshter R, Hardemann J, Brodsky M Magnesium and potassium therapy in multifocal atrial Magnesium and potassium therapy in muldif

22 Kulick D, Hong R, Ryzen E, et al. Electrophysiologic effects of intravenous magnesium in patients with normal conduction systems and no clinical evidence of significant cardiac disease. Am Heart $\mathcal{F} 1988 ; 115: 367-73$.

23 Rasmussen H, Thomsen P. The electrophysiological effects of intravenous magnesium on human sinus node, atrioventricular node, atrium, and ventricle. Clin Cardiol 1989;12:85-90.

24 Rogiers P, Vermeier W, Kesteloot H. Effect of the infusion of magnesium sulfate during atrial pacing on ECG and blood pressure. $A m$ Heart $\mathcal{F}$ 1989;117:1278-83.

25 Perticone F, Ceravolo R, Costa R, Mattioli P. Electrophysiologic effects of magnesium sulfate infusion in patients with cardiac conduction defects. $₹ \mathrm{Am}$ Coll in patients with cardiac

26 DiCarlo L, Morady F, De Buitleir M, Krol R, Schurig L Annesley $T$. Effects of magnesium sulfate on cardiac Annesley T. Effects of magnesium sulfate on cardiac Conduction and refracto $1986 ; 7: 1356-62$.

27 White R, Hartzell H. Magnesium ions in cardiac function Regulator of ion channels and second messengers. Biochem Pharmacol 1989;38:859-67.

28 England $M$, Gordon G, Salem M, Chernow B Magnesium administration and dysrhythmias after cardiac surgery. A placebo-controlled, double-blind, randomized trial. $\mathcal{F A M A}$ 1992;268:2395-402.

29 Davidenko J, Cohen L, Goodrow R, Antzelevitch C. Ouinidine-induced action potential prolongation, early after depolarizations, and triggered activity in canine Purkinje fibers. Circulation 1989;79:674-86. 